\title{
What's English Majors' View on the Best Time of Second Language Learning?
}

\author{
Yuchen Shang \\ School of International Studies, Zhejiang University, Hangzhou, Zhejiang, China
}

\begin{abstract}
Based on relevant theories in the past, this article explores the best time for English majors to learn a second language from the perspective of research methods and data analysis, with a view to providing strategies and references for relevant language learners.
\end{abstract}

Keywords: English majors; second language; best time

\section{Introduction}

The best time of second language learning is the most suitable, effective and easiest time for one to achieve a new kind of language except his or her native language. Currently, it has become a hot topic in that citizens gradually realize the significance of acquiring a second language themselves and the language education of their children. (Yang, 1998) As English majors, who tend to be relatively more professional in second language acquisition, may have a special kind of view on this topic. Hence, their ideas on this topic are worth researching.

In this time and age, with the rapid development of English mania all around China, the time children begin to learn a second language become earlier and earlier. (Liu, 2016) However, it is not the fact that the earlier children get exposed to a second language, the better they are able to learn. One thing is that children may be familiar with the wrong pronunciation if their teachers' teaching level are blow the average. The other is that children are commonly less interested in learning language itself, which may generate less motivation.

The best time for one to learn a second language has already been researched by some studies. Among them on this question, critical period hypothesis is of great influence. Namely, after adolescence, lateralization happens in most humans' brain, indicating that learning a second language become more and more difficult. (Hu, 2013)

Therefore, this research tries to figure out English major's view on the best time of second language learning as well as the factors leading to it.

\section{Literature Review}

Since the best time of second language learning has been one of the hottest concern in this time and age, plenty of researches are able to be found which aim at studying and discussing the most suitable time of second language acquisition. A majority of researchers believed in critical period hypothesis raised by Lenneberg indicating that the time before the end of puberty is the best time for one to acquire a second language. (Ye, 2012) While others call into question the idea, holding that it is not always the truth that learning second language as early as possible is the most appropriate choice. (Yang, 2012; Li, 2016) Meanwhile, researches about English majors' view on this topic are instructive but not complete.

Copyright (C) 2020 by author(s) and Frontier Scientific Research Publishing Inc.

This work is licensed under the Creative Commons Attribution International License (CC BY 4.0).

http://creativecommons.org/licenses/by/4.0/ 
Based on researches about the question of how language represented in the human brain in the realm of neuroscience and neurolinguistics, neurophysiologist Penfield and Roberts demonstrates that learning second language in critical period is the most suitable and effective strategy in education. (Cohen, 1998) After two years old, in the process of lateralization, second language acquisition gradually encounters more and more obstacles. (Krashen,1981) This theory has aroused strong repercussions in linguistic circles since it was put forward. Furthermore, some researchers have conducted a functional magnetic resonance imaging (fMRI) study to examine the neural activities associated with second language word learning to prove it. (Jing, Kathleen, 2015; Edna, 2013) Although it still remains at the hypothetical level, it has been regarded as a golden rule by many English teachers and parents in China. The public has commonly shifted the expecting age in English learning, foreign language learners becoming younger and younger.

In terms of the acquisition of language, some scholars regard language as a culture phenomenon as well as a neurological one, showing that learning second language as early as possible may contribute much to its acquisition. They believe that the process of children's language learning process can be divided into three parts - preparation (1-1.5years old), development of spoken language (1.5-3 years old) and development of written language (3-6 years old). (Edna Andrews, 2013) From those mentioned above, supportive researchers announced that learning second language before seven will meet optimum results. (Xiao, 1993) Some researchers claimed that the process and results of learning a second language are straightly connected with the acquisition of the first language. (Stu Silverberg, 2004) Adults learn second language with much more linguistic habits and thinking styles coming from their first language. On the contrary, children commonly learn second language as natural as the first one. (Dai, 2006) To some extent, learning second language at a young age made learning itself an easy job.

Although plenty of researchers are in favor of the critical period hypothesis, dissenting voices still exist, stating that it is not necessary to learn second language at a very young age. Some hold that neuropsychological studies further showed that lateralization has been completed before puberty. Hence, they came out with a sensitive period hypothesis which is relatively eclectic - it is meaningful to get exposed to second language at young age for the reason that it helps stimulate the development of related nervous function system (Yang, 1998) Some scholars claimed that age has little effect on language learning in that there is more than one critic period which outranges from two to twelve and there are other factors that play a key role in language studying. (Nicloe, 2014; Muhammad, 2015; Wang, 2001) Krashen, a linguist, strongly opposes the critical period theory, arguing that the differences in language acquisition between children and adults are not caused by the language mechanism itself, but by the external factors of the language mechanism. The difference of comprehensible input and the enhancement of affective filter ability result in the difference of language acquisition between children and adults. (Krashen,1981; Hu, 2013) Age is not the preheater of language acquisition success.

The above description reminds us that, with optimal and large input, learners of any age can make progress in language proficiency, even close to the level of native speakers. Meanwhile, time and economic condition permitted, getting exposed to second language properly at young age may be conducive to second language acquisition for the reason that kid's brain may be sensitive to language. Nevertheless, researches about English majors' view on the best time of second language learning are not sufficient enough. Commonly, English majors are the only groups of people who are required to learn a second foreign language in school. Hence, strategies used by English majors to learn second language are instructive to the study of the best time of second language acquisition. (Zhong, 2017)

\section{Research Method}

This research intends to study on English majors' view on the best time of second language learning and the relationship between starting age and language learning. Hence, a survey with online questionnaires was designed and 
conducted to collect English majors' views and their performance in language learning with an online questionnaire platform "WJX". A total number of one hundred participants (English majors of Zhejiang University) took part in this survey, more than a half (51\%) of which are sophomores. While there are $17 \%$ freshmen, $17 \%$ junior students and $15 \%$ senior students. Each student who finished the questionnaire will be given a WeChat red pocket. After that, an online mathematical statistics analysis platform SPSSAU together with EXCELL were used to deal with the answers collected from the survey. This section mainly focuses on the data collection methods, sampling and data analysis strategies.

Participants were given 22 questions consisting of two types, relationship measuring questions (between starting time and academic performance) and view collecting questions. Multiple choices, gap filling questions and 5-point scale questions are included to analyze English majors' views on the best time of language acquisition and the relationship between their starting time and performance in language learning. Multiple choices and 5-point scale questions are used to value English majors' all-around English learning effect in specific, and then make a comparison between it and the time they start to learn. While gap filling choices like "Why do you suggest learning English before/after the age of 6?" are provided as open questions to find out English majors' personal feelings about their ideas towards the best starting time to learn a language and the degree of satisfaction towards their own learning process.

Relationship measuring questions are designed based on the Strategy Inventory for Language Learning (SILL) standard questionnaire in order to value English majors' real language learning level (Bachman L., 1990). Nevertheless, there are totally 50 questions in SILL, which is relatively complicated for testees and may make them lose the interest when finishing the questionnaire. Therefore, to make the survey simple but effective, questions like "What kind of strategy do you prefer when learning English?" are designed as assessment on the rationality of their language learning methods. Commonly speaking, the more rational learning strategy one has, the better results of language learning one will get. Thus, analysis towards the rationality of language learning method contributes to the research of one's language learning level. At the same time, 5-point scale questions like "How do you evaluate your English writing/ listening/ reading/ speaking ability?" are added, to measure the English learning level by providing scores ranging from 1 to 5 . Then scores are collected in each dimension (English writing/ listening/ reading/ speaking) to form a comprehensive score of language learning level, which will be compared with the starting time of language learning in order to analyze the correlation between them and find out whether the earlier means the better.

View collecting questions consist of multiple choices and open questions, aiming at collecting English majors' view on the best time of language learning intuitively and comprehensively. Questions like "Are you in favor of learning English before 6 years old?" are given and the answer should be chosen from "A: Yes." and "B: No." for the sake of the ocular of information. Besides, English majors are required to give their personal reasons for their choice, which may make up the essential source of the discussion of English majors' view on the best time of second language learning.

What's more, in order to find out whether there are other factors that have a more significant influence than age on language learning, which is mentioned by those who are against critical period hypothesis, questions concerning parents' English level, interest of the language and the preference of learning strategies are set up in the questionnaire. For instance, "Do you enjoy learning English?" is given as one of the questions and choices provided are "A: I have keen interest in it.; B :I often prefer learning it. C: Just so so. D: I have no interest in it."

\section{Data Analysis}

This section focuses on the analyzing process and the findings of the statistics collected from the survey. Above all, the questions and answers are classified as relationship measuring questions and view collecting questions. Afterwards, to test whether there is a strong correlation between the start time of language learning and the performance after learning, 
Pearson's correlation coefficient is used to analyze statistics collected from the survey.

Pearson's correlation coefficient when applied to a population is commonly represented by the Greek letter $\rho$ (rho) and may be referred to as the population correlation coefficient or the population Pearson correlation coefficient. Given a pair of random variables $(\mathrm{X}, \mathrm{Y})$, the formula for $\rho$ is:

$$
\rho_{X_{r} Y}=\frac{\operatorname{cov}(X, Y)}{\sigma_{X} \sigma_{Y}} \quad r=\frac{1}{n-1} \sum_{i=1}^{n}\left(\frac{X_{i}-\bar{X}}{\sigma_{X}}\right)\left(\frac{Y_{i}-\bar{Y}}{\sigma_{Y}}\right)
$$

( $\operatorname{Cov}$ is the covariance; $\sigma \mathrm{x}$ is the standard deviation of $\mathrm{X}$; $\sigma \mathrm{y}$ is the standard deviation of $\mathrm{Y} ; X_{i}, \bar{X}$ are defined as above, and $\sigma_{X}$ are defined below; $\frac{X_{i}-\bar{X}}{\sigma_{X}}$ is the standard score and analogously for the standard score of y.)

This correlation coefficient is conducive to estimate the correlation level between language learning performance and factors that may have an influence on it by the absolute value $|\mathrm{r}|$. In the above-mentioned formula, $|\mathrm{r}| \approx 0.5$ demonstrates a relatively strong correlation and $|\mathrm{r}| \approx 0.3$ indicates a medium strong correlation, while the correlation is weak when $|\mathrm{r}|$ is less than 0.2. In other words, the more $|\mathrm{r}|$ reaches 1 , the stronger the correlation between the two variables.

After organizing and analyzing the data, we initially have a general idea of English majors' view on the best time of language learning. There are totally 100 students who take part in this survey, and 79 of them support that human beings should acquire language before the age of six. That is to say, a majority of English majors believe that the best time for language learning is before primary school.

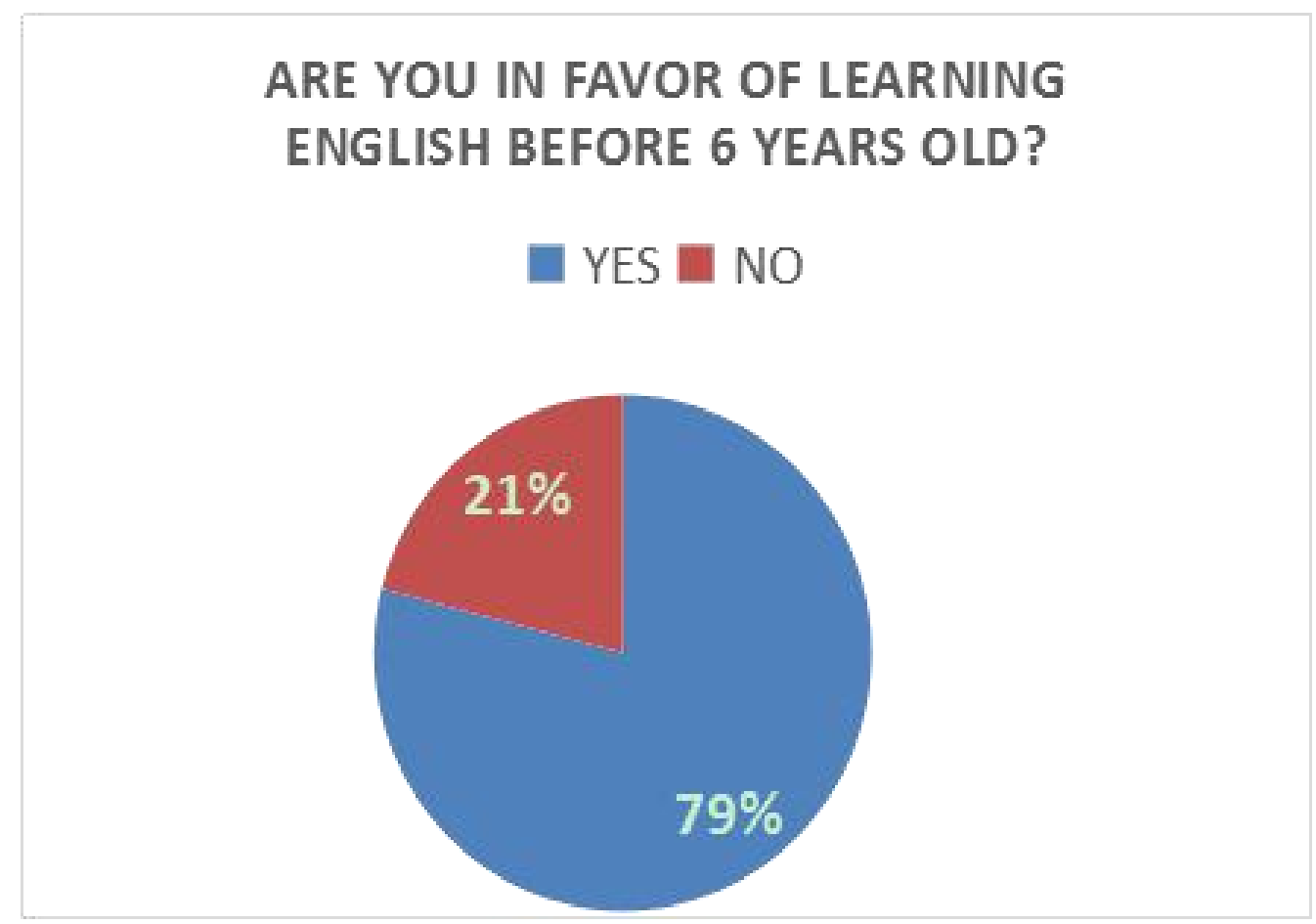

Figure 1. Whether English majors support learning English before the age of 6 or not

However, in regard to the next question "What do you think is the most suitable age to start learning English?", when English majors are given more choices like "A: 1-5. B: 6-10. C: 11-15. D: 16-20. E: After 20." What is kind of astonishing is that a half of them chose "B: 6-10.", while $48 \%$ of them chose "A: 1-5.", which is a bit different from the findings of the last question. The different results indicate that there are some hesitation and uncertainty in English majors' mind about whether getting exposed to a second language is completely beneficial to kids. 


\section{WHAT DO YOU THINK IS THE MOST SUITABLE AGE TO START LEARNING ENGLISH?}

$1-5$ years old $\mathbf{E} 6-10$ years old $\mathbf{E}$ 11-15 years old $\mathbf{E} 16-20$ years old $\mathbf{a}$ after 20 years old

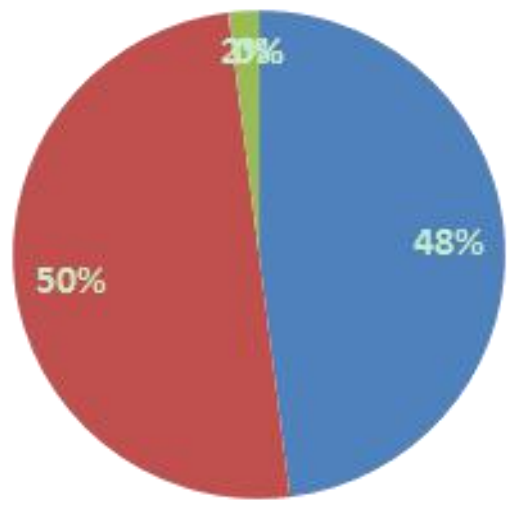

Figure 2. English majors' view about the best time to start learning a second language

After that, Pearson's correlation coefficient is used to analyze several factors that may have an influence on second language learning. In chart 3, given the $|r|$ calculated from two groups of the statistics (the starting time of second language learning and the performance of English writing/ listening/ reading/ speaking), it is vivid that the second language performance is relative to the start time of learning. For instance, the correlation $(|\mathrm{r}|)$ between the time to begin learning English and their English listening performance is 0.254 and shows a statistical significance of 0.05 , which indicates that there is a significant positive correlation between the beginning time and English listening performance.

Table 1. Correlation between the starting time and second language performance

\begin{tabular}{|c|c|c|c|c|}
\hline & $\mathrm{p}$ & $|\mathrm{r}|$ & Correlation & Degree \\
\hline Listening & 0.011 & 0.254 & positive & Medium \\
\hline Reading & 0.007 & 0.269 & positive & Medium \\
\hline Writing & 0.009 & 0.258 & positive & Medium \\
\hline Speaking & 0.001 & 0.316 & positive & Strong \\
\hline
\end{tabular}

The degree of learning difficulty, to some extent, is an indication of students' language learning situation. After comparing the statistics, we surprisingly find that the $21 \%$ of the testees who regard learning English a hard job is somehow coincide with the late beginners. The $|r|$ in this correlation being -0.347 suggests a negative strong correlation between the starting time and the difficulty when learning English.

English. Hence, the earlier one starts to learn English, the easier he feels during the process of language acquisition.

Table 2. Correlation between the starting time and degree of learning difficulty

\begin{tabular}{|c|c|c|}
\hline Options & Number of People & Proportions \\
\hline Difficult & 23 & $23 \%$ \\
\hline Ordinary & 56 & $56 \%$ \\
\hline Easy & 21 & $21 \%$ \\
\hline Sum & 100 & \\
\hline
\end{tabular}


Other potential influencing factors consisting of parents' English level, interest of the second language and the preference of learning strategies are also vital to the research of the best time of second language learning. We analyze those factors by the formula of Pearson's correlation coefficient and finally find that the results show great contingency in that the correlation between parents' English level and the language performance is fairly weak, while the correlation between interest and performance is rather high. Nevertheless, although uncertainty is always there, risk accompanying decisions, the result still leaves us plenty of inspirations worthy of consideration.

Table 3. Correlation between the second language performance and other potential influencing factors

\begin{tabular}{|c|c|c|c|c|}
\hline & $\mathrm{p}$ & $|r|$ & Correlation & Degree \\
\hline Parents' English level & 0.533 & -0.063 & Negative & Weak \\
\hline Interest of the second language & 0.000 & -0.506 & Negative & Strong \\
\hline
\end{tabular}

\section{Discussion}

Based on the above-mentioned data analysis and the results, we describe English majors' view on the best time of second language learning. Our studies establish the idea that children should begin learning a second language as earlier as possible.

According to the survey, generally, more students are in favor of the view that the best time for second language learning is the time before the end of puberty, which is in accordance with the critical period hypothesis being mentioned in the previous literature. The correlation analysis towards the beginning time and language learning performance demonstrates that the second language performance is relative to the start time of learning, since the $|\mathrm{r}|$ of English writing, reading, listening or speaking is more than 0.2 . Besides, due to the data of the correlation test between the starting time and degree of learning difficulty, it is vivid that the earlier kids start to learn a new language, the easier they will find it would be.

What's more, the correlation analysis between the second language performance and other possible influencing factors proves that although there may be several factors which is relative to the second language performance, the effect of the beginning time of language learning is always dominant.

Meanwhile, in spite of several discoveries revealed by these studies, there are also limitations. Because there are only one hundred samples in the survey, English majors' choices being likely to be influenced by the surroundings, it is not surprised that the data and final results show great contingency and uncertainty. Besides, out of the restriction of the questionnaire, it is hard to testify whether learning a second language too earlier does harm to kids, like decreasing their interest in the language itself.

\section{Conclusions}

Our findings confirm that English majors' view on the best time of second language learning is before the end of puberty, after which language acquisition gradually encounters more and more obstacles. Although there still exists some dissenting voices in the previous literature, stating that the influence of age may be exceeded by other factors like their parents' language level, the influence of age towards the language learning is always the dominance based on the statistics collected from the survey and the analysis.

By all counts, the study thus offers a strategy for language learning that if condition permitted, it is advisable for kids to learn a second language as earlier as possible. 


\section{Conflicts of Interest}

The author declares no conflicts of interest regarding the publication of this paper.

\section{References}

[1] Bachman L. (1990). Fundamental Considerations in Language Testing. Oxford: Oxford University Press.

[2] Cohen A.D. (1998). Strategies in Learning and Using a Second Language. London: Longman.

[3] Chen Z.Y. (2012). Critical Period Hypothesis and Second Language Education. Journal of Changchun University of Technology, (7): 155-156.

[4] Cui X.L. (2014). Reflections on Language Education. Young and Middle-aged Learners of Linguistics Salon.

[5] Chen J.Y. (2015). The Neural Mechanism Affecting Second Language Learning and Its Enlightenment to Second Language Education. Global Education, (11):121-128.

[6] Dai M.C. (1998). UG Theory and Second Language Acquisition Research. Foreign Language and Foreign Language Teaching, (2):5.

[7] Elis Rod. (1994). The Study of Second Language Acquisition. Oxford: Oxford University.

[8] Edna Andrews. (2013). Multilingualism and fMRI: Longitudinal Study of Second Language Acquisition. Brain Sciences, 3(2): 849-876.

[9] Fu H.X. (2009). Enlightenment of Mother Tongue Acquisition on Foreign Language Learning. Journal of Jiangsu Vocational and Technical College of Architecture, 9(1): 62-63.

[10] Hu G.M. (2013). Advantages and Disadvantages of Learning English in Childhood. Journal of Jiangsu Vocational and Technical College of Architecture, (2): 84-86.

[11] Jing Y. (2015). Kathleen Marie Gates, Neural Changes Underlying Successful Second Language Word Learning: An fMRI Study. Journal of Neurolinguistics, (33): 29-49 .

[12] Krashen S. (1988). Second Language Acquisition and Second Language Learning. Prentice Hall.

[13] Li H.T. (2011). Activity Levels in The Left Hemisphere Caudate-fusiform Circuit Predict How Well A Second Language Will be Learned. PNAS, (108): 2540-2544.

[14] Li X.H. (2016). Rethinking about English Education for Children. English Square, (71): 151-152

[15] Muhammad Asif Qureshi. (2016). A Meta-analysis: Age and Second Language Grammar Acquisition. System, (06): 147-160.

[16] N. Nau. (2014). First and Second Language Acquisition: Towards a Reconcillation. Lingua Posnaniensis, (56):125-140.

[17] Stu Silverberg and Arthur G. Samuel. (2004). The Effect of Age of Second Language Acquisition on the Representation and Processing of Second Language Words. Journal of Memory and Language, (51):381-398.

[18] Sara Ramos. (2017). Does Learning a Language in the Elderly Enhance Switching Ability. Journal of Neurolinguistics, (43): 39-48.

[19] Shekeila D. Palmer. (2010). Age of Acquisition Effects in Vocabulary Learning. Acta Psychologica, (135): 310315 .

[20] Wang L.F. (2001). Second Language Learning Strategies: Problems and Solutions. Foreign Language Teaching (FLTA), (4): 5-13.

[21] Xie S.T. (2012). Effects of Age and Learning Time on English Phonetics. Journal of Hubei Second Normal University, (9): 84-87.

[22] Xiao P. (1993). Critical Age and Education for Bilingual Formation of Children. Psychological Development and 
Education, (4): 32-35.

[23] Yang L.R. and Cui P. (2012). Reflections on the Critical Period Hypothesis in the Debate. Foreign Language Teaching Theory and Practice (FLLTP), (3): 43-49.

[24] Yang L.R. (1998). On Age in Foreign Language Teaching. Foreign Language Teaching, (2): 35-40.

[25] Ye H. (2012). On the Necessity of Opening English Courses in Kindergartens. Primary and Secondary Education, (121): 265-266.

[26] Zhong Z.H. (2017). A Survey of the Transfer Role of First Language Learning Strategies of College English Majors in Second Language Learning. Journal of Inner Mongolia Normal University, (8): 121-124. 